\title{
The influence of secondary hyperparathyroidism -at the time of index operation- on the later development of (septic or aseptic) loosening of implants in female patients with knee osteoarthritis who undergo Total Knee Arthroplasty
}

\author{
Kyriakos A. Papavasiliou, Theodore Michael, Marinos Th. Karanassos, \\ Dimosthenes Tsitouras, John M. Kirkos
}

\author{
$3^{\text {rd }}$ Orthopaedic Department, Aristotle University of Thessaloniki Medical School, \\ Papageorgiou General Hospital, Thessaloniki, Greece.
}

Introduction: Parathyroid hormone (PTH) is a major regulator of bone metabolism and calcium homeostasis. Secondary hyperparathyroidism ( $\mathrm{SH}$ ) manifests as a result of decreased calcium intake, impaired intestinal absorption of calcium due to ageing or disease, vitamin-D deficiency, impaired renal function with diminished 1a-hydroxylase enzyme availability, or parathyroid gland resistance to $1,25(\mathrm{OH})^{2} \mathrm{D}$-facilitated inhibition of PTH secretion. It is a 'silent epidemic' among postmenopausal women-the most common group undergoing total knee replacement (TKR). Continuously elevated PTH levels activate osteoclasts; intermittent administration of PTH induces osteoblastic activity and may enhance early fixation of both cemented and non-cemented implants. Fixation of an implant depends partly on bone growth at its surface, and may be the end result of fracture healing. The actual effects (if any) on the implant incorporation process by continuously elevated serum PTH levels remain unknown.

Objectives: Aim of this prospective casecontrolled study was to assess whether the incidence of loosening of implants in patients undergoing Total Knee Arthroplasty (TKA) is higher among those with Secondary Hyperparathyroidism (SP) at the time of index operation.

Methods: Caucasian female patients with knee osteoarthritis who underwent TKA between November 2004 and March 2007 were enrolled. Exclusion criteria: endocrinopathies, rheumatoid or secondary arthritis, renal disease, fracture or orthopaedic surgery 24 months before enrollment. Case-notes were reviewed for any revision operation.
Patients with osteopenia/osteoporosis were not excluded. Serum intact PTH (I-PTH), calcium, phosphorus, creatinine, and the clearance of creatinine were determined pre-operatively.

Results: 283 women aged 49 to 81 (mean 70) were enrolled; 100 had abnormally elevated IPTH. Two with primary hyperparathyroidism were excluded from analysis. The incidence of $\mathrm{SH}$ was $35 \%$. I-PTH correlated positively with age $(p=0.008)$ and creatinine level $(p=0.021)$ and negatively with the clearance of creatinine $(p=0.004)$. In multiple regression analysis, $7.3 \%$ of the variance in I-PTH values $\left(R^{2}=0.073, p<0.001\right)$ was significant; creatinine level was the largest contributor (standardised beta=0.275, $p=0.08$ ). 265 patients were available for re-evaluation at an average followup period of 115 (105-132) months. Three patients with $\mathrm{SH}$ and 7 with normal I-PTH values (at the index operation) were reoperated due to aseptic loosening at an average period of $51.3(26-90)$ and 49.9 (2594) months respectively. The difference between the number of re-operated patients with pre-operative $\mathrm{SH}$ and normal I-PTH values, was statistically non-significant ( $p>0.005)$. The mean time to re-operation was also non-significant $(p>0.005)$. One patient with $\mathrm{SH}$ and two with normal I-PTH, were reoperated due to septic loosening ( $p>0.005)$. The mean time to revision operation was 7.6 (4-12) months.

Conclusions: Our results show that $\mathrm{SH}$ does not enhance aseptic loosening of implants in TKA. Larger series are needed, especially as far as septic loosening and $\mathrm{SH}$ is concerned.

Conflict of Interest. The authors declare no Conflict of Interest regarding the preparation of this paper.

\section{References}

1. Papavasiliou KA, Kenanidis EI, Potoupnis ME, Sarris IK, Kirkos JM, Kapetanos GA. Incidence of secondary hyperparathyroidism among postmenopausal women with end-stage knee osteoarthritis. J Orthop Surg (Hong Kong) 2009;17(3):310-2.

2. Papavasiliou KA, Nikopoulou A, Kenanidis El, Potoupnis ME, Kyrkos MJ, Sayegh FE, Kapetanos GA. Serum intact-parathyroid hormone level following total knee arthroplasty. J Orthop Surg (Hong Kong). 2012;20(1):27-31 